 \\ Tunable and multiple-wavelengths/temporal output from gain-switched diode laser and a four Bragg-grating Fiber
}

Cite as: Appl. Phys. Lett. 85, 861 (2004); https://doi.org/10.1063/1.1779350

Submitted: 14 October 2003 . Accepted: 09 June 2004 . Published Online: 04 August 2004

E. U. Rafailov, I. G. Cormack, F. Floreani, L. Zhang, I. Bennion, and W. Sibbett

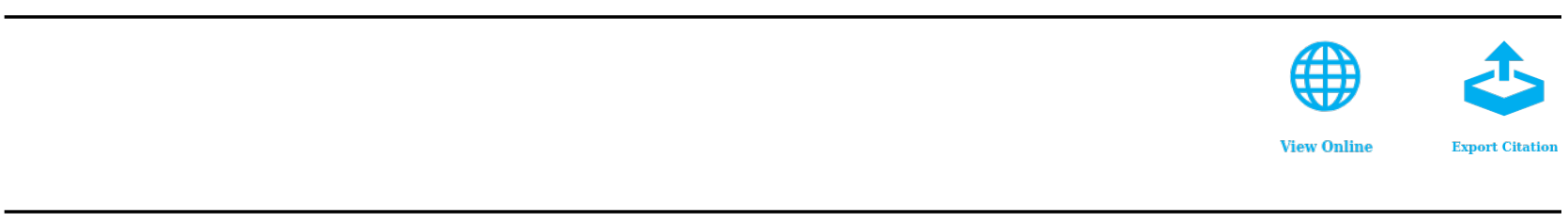

\section{Applied Physics Reviews}

Now accepting original research 


\title{
Tunable and multiple-wavelengths/temporal output from gain-switched diode laser and a four Bragg-grating Fiber
}

\author{
E. U. Rafailov ${ }^{\text {a) }}$ \\ School of Physics and Astronomy, University of St. Andrews, North Haugh, St. Andrews, Fife KY16 9SS, \\ United Kingdom \\ I. G. Cormack \\ ICFO_Institut de Ciencies Fotoniques, Jordi Girona 29, Nexus II, 08034 Barcelona, Spain \\ F. Floreani, L. Zhang, and I. Bennion \\ Electronics Engineering and Applied Science, Aston University, Birmingham B4 7ET, United Kingdom \\ W. Sibbett \\ School of Physics and Astronomy, University of St. Andrews, North Haugh, St. Andrews, Fife KY16 9SS, \\ United Kingdom
}

(Received 14 October 2003; accepted 9 June 2004)

\begin{abstract}
We demonstrate spectral narrowing and selective tuning of the picosecond pulse outputs from an injection-seeded, gain-switched diode laser that is simultaneously coupled to four output fibers with different Bragg-grating resonators. A single-, dual-, and triple-wavelength and temporal pulse operation from all four outputs is presented. (C) 2004 American Institute of Physics.
\end{abstract}

[DOI: $10.1063 / 1.1779350]$

A compact laser source that is capable of simultaneously generating a multiple number of tunable, near transformlimited picosecond pulses is of great interest to numerous scientific and practical applications such as wavelengthdivision-multiplexed communication systems, optical sensors and switches, optical signal processing and optically pumped mm-wave generators. Dual-wavelength operation has been achieved for mode-locked laser diodes with different configurations ${ }^{1,2}$ as well as for gain-switched diode lasers. ${ }^{3,4}$ In this letter we demonstrate spectral narrowing, selective wavelength tuning, and multiple picosecond pulses at four separate, discrete wavelengths from a single gainswitched diode laser that is inserted into an optical fiber from which the light is coupled into four different Bragg grating outputs. ${ }^{5}$ The individual reflectivities of these Bragg gratings determine the wavelengths that are able to be emitted from the laser. The specific selection of these wavelengths is dependent upon the externally applied rf frequency of the gainswitched laser diode laser.

A commercial InGaAs/GaAs single-mode ridge waveguide laser with an active stripe width of $3 \mu \mathrm{m}$ and an emission wavelength of $980 \mathrm{~nm}$ was used. The typical gainswitched pulse duration without optical feedback was $\sim 35 \mathrm{ps}$ and had a pulse repetition frequency between 1.80 and $2.70 \mathrm{GHz}$. An average power of $150 \mathrm{~mW}$ (peak power $\sim 1.5 \mathrm{~W}$ ) was achieved with pulses having spectral bandwidths up to $20 \mathrm{~nm} .^{6}$

The fiber fabricated for this work was designed to provide spectral narrowing and a multiple wavelength/temporal output. By using a chirped phase mask as a 50/50 beamsplitter within a Talbot interferometer ${ }^{7}$ it was possible to write a Bragg grating within a hydrogen-loaded $980 \mathrm{~nm}$ wavelength single mode fiber from Fibercore. By varying the distance between the phase mask and the fiber it was pos-

${ }^{a)}$ Electronic mail: er8@st-andrews.ac.uk sible to provide accurate control over the period of the grating.

The four gratings were designed to reflect at four separate wavelengths $(973,978,981$, and $993 \mathrm{~nm})$. From measurements using a white light source and an optical spectrum analyzer, it was found that three of the four gratings had similar reflectivities around 5-6.5 dB $(64 \%-73 \%)$ and the fourth grating at $993 \mathrm{~nm}$ was found to have a lower reflectivity $\sim 2.9 \mathrm{~dB}(47 \%)$. This reflectivity difference is important to note as it plays an important role in the ease at which differing wavelengths can be observed.

Figure 1 shows a simplified scheme of our experiment. The four output fibers, each having a Bragg-grating of different period, were spliced to a $1 \times 4$ coupler with an inputport. The laser output was initially butt coupled into a- piece of unprocessed fiber (ends were not specially treated). The power transmitted through the fiber grating was dependent upon the rf power and frequency. For a current of $180 \mathrm{~mA}$ the maximum transmission out of each of the four output fibers was $\sim 7.5 \mathrm{~mW}$. It should be noted that the fiber ends were simply cleaved and consequently the optical coupling was not optimized. Under nonresonant injection-seeded condition $^{8}$ all outputs ends could emit single wavelength

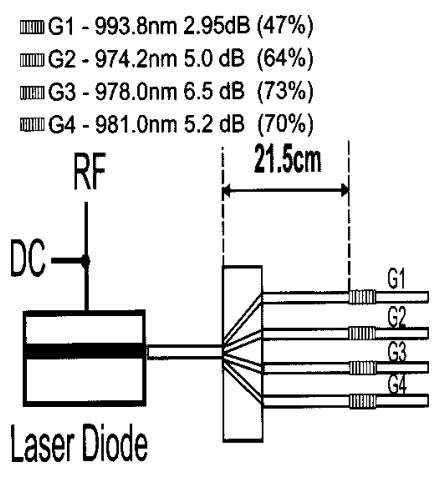

FIG. 1. Simplified optical scheme. 
(a)

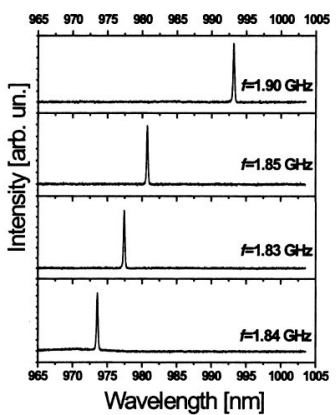

(b)

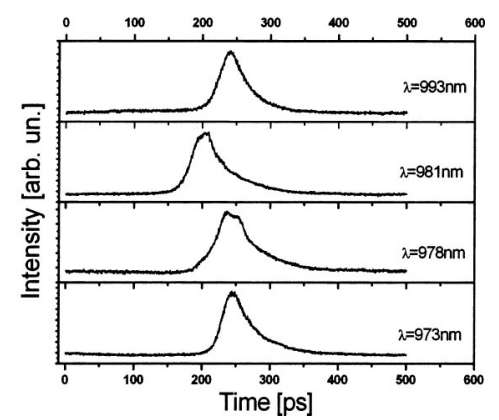

FIG. 2. (a) Spectra characteristics for single-wavelength output cases; (b) corresponding pulse shapes.

973, 978, 981, or $993 \mathrm{~nm}$ such as presented in Fig. 2(a). (Nonresonant condition is the round-trip period of the external cavity slightly detuned from the rf modulation period and its harmonics.) The emitted wavelength was dependent upon which Bragg grating was dominating at the supplied rf fre-

(a)

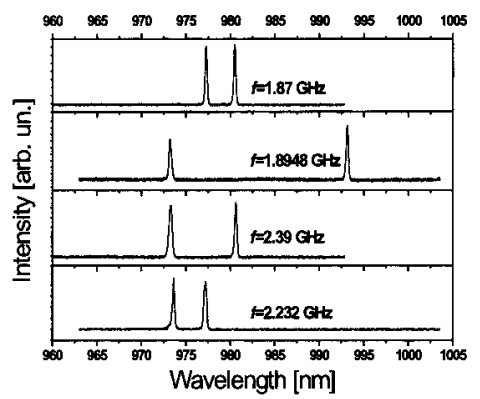

(b)

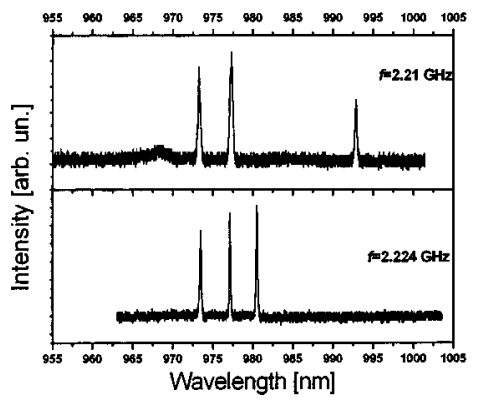

FIG. 3. (a) Spectra characteristics for dual-wavelength output cases; (b) spectra characteristics for triple-wavelength output cases.

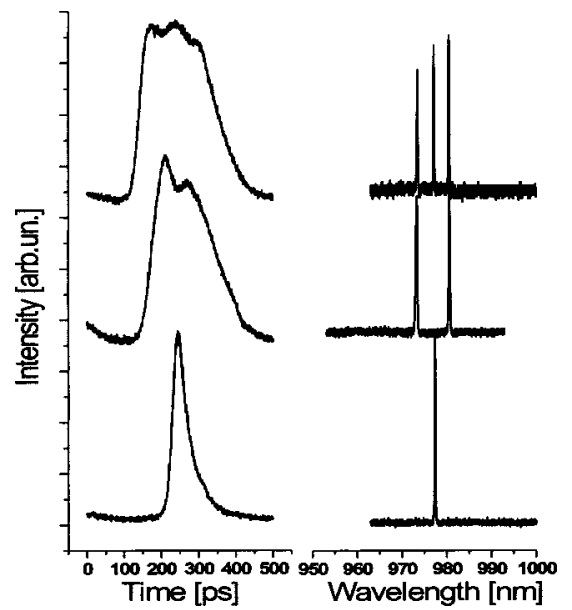

FIG. 4. Temporal (left) and spectral (right) characteristics for single-, dual-, and triple-wavelength outputs.

quency. Figure 2(b) presents their corresponding pulse shapes.

It was also possible to obtain from all output fibers dual and triple wavelengths simultaneously [see Figs. 3(a) and 3(b)]. It is worth pointing out that an output of any combination of multiple wavelengths is only produced at modulation frequencies that satisfy the nonresonant conditions for all cavities arms simultaneously (including harmonics). Figure 4 shows temporal characteristics for single-, dual-, and triple-wavelength outputs. The temporal position of each pulse is dependent upon the path difference between each grating. By deliberately creating specific path differences between each grating the temporal positions of each pulse can be selected individually.

As previously mentioned, the reflectivity from one of our gratings (G1) was two times smaller than the other three gratings. We believe that this was the reason why we did not observe four wavelengths simultaneously.

In conclusion we demonstrate that a nonresonant injection-seeding technique applied to gain-switched diode laser allows one to obtain spectral narrowing and selective tuning of the picosecond pulse outputs from a single laser diode using a fiber with four Bragg-grating outputs. A single-, dual-, and triple-wavelength and temporal pulse operation from all four outputs is presented.

${ }^{1}$ B. Chu and I. H. White, Appl. Phys. Lett. 65, 2928 (1994).

${ }^{2}$ D. Burn, G. Hay, and W. Sibbett, Tech. Digest, CLEO, Baltimore, MD, 444, JThA3, 1993.

${ }^{3}$ C. Shu and Y. H. Lee, IEEE J. Quantum Electron. 32, 1976 (1996).

${ }^{4}$ D. N. Wang and C. Shu, IEEE Photonics Technol. Lett. 9, 1211 (1997).

${ }^{5}$ I. G. Cormack, E. U. Rafailov, W. Sibbett, F. Floreani, L. Zhang, and I. Bennion, CLEO/Europe'03, Munich, Germany, CC8-5-WED, 2003.

${ }^{6}$ D. J. L. Birkin, E. U. Rafailov, and W. Sibbett, Appl. Phys. Lett. 11, 1862 (2002).

${ }^{7}$ F. Floreani, A. Gillooly, D. Zhao, L. Zhang, I. Bennion, X. Shu, and K. Sugden, J. Opt. A, Pure Appl. Opt. 5, 59 (2003).

${ }^{8}$ E. U. Rafailov, D. J. L. Birkin, W. Sibbett, E. A. Avrutin, IEEE J. Sel. Top. Quantum Electron. 7, 287 (2001). 